\title{
Prevalencia de periodontitis crónica moderada y avanzada generalizada como factor de riesgo cardiovascular.
}

\author{
Prevalence of chronic periodontitis moderate and advanced \\ generalized as a cardiovascular risk factor.
}

Keyla Esmeralda Martínez Benítez,* Rosa María Bulnes López, ${ }^{\ddagger}$ Mónica González Alemán

\section{RESUMEN}

Introducción: La periodontitis es una enfermedad infecciosa que afecta a los tejidos de sostén de los dientes, su prevalencia se estima entre el 35 y 45\% de la población adulta. Las enfermedades cardiovasculares tienen relación directa con la periodontitis crónica, las bacterias periodontales pueden pasar la barrera epitelial de los tejidos periodontales y lograr la propagación sistémica a través de los vasos sanguíneos, causando la inflamación del endocardio. Objetivo: Determinar prevalencia de enfermedad periodontal como factor de riesgo cardiovascular en adultos de 25 a 60 años en Nacajuca, Tabasco. Material y métodos: Se realizó un estudió observacional, prospectivo, transversal y analítico, con muestra de 40 participantes entre 25 y 60 años, el 52\% (21) son hombres y el $49 \%$ (19) mujeres, se empleó el sistema no probabilístico por conveniencia. Las variables fueron edad, género, grado de conocimiento sobre enfermedad periodontal, nivel de alimentación, grado de tabaquismo, grado de higiene bucal y grado de periodontitis. Resultado: La prevalencia de enfermedad periodontal como factor de riesgo cardiovascular es $48 \%$ (19 personas) con periodontitis crónica moderada y avanzada generalizada. Conclusión: Con base en la revisión bibliográfica, las personas con el grado de periodontitis crónica moderada y avanzada generalizada son propensas a desencadenar una enfermedad cardiovascular (infarto agudo de miocardio). Sin embargo, son necesarios más estudios de experimentación longitudinal, con base en el vínculo que tiene la enfermedad periodontal y cardiovascular.

Palabras clave: Enfermedad cardiovascular, infarto agudo de miocardio, periodontitis crónica.

\section{ABSTRACT}

Introduction: Periodontitis is an infectious disease that affects the tissue of the teeth, its prevalence is estimated between 35 and 45\% of the adult population. Cardiovascular diseases are directly related to chronic periodontitis, periodontal bacteria can pass the epithelial barrier of periodontal tissues and achieve systemic propagation through the blood vessels causing Inflammation of the endocardio. Objective: To determine the prevalence of periodontal disease as a cardiovascular risk factor in adults from 25 to 60 years Nacajuca, Tabasco. Material and methods: An observational, prospective, transverse and analytical study was carried out, with a sample of 40 participants between 25 and 60 years, 52\% (21) corresponds to the masculine genus and 49\% (19) represents the female genus, the non-probabilistic system was employed by Convenience. The variables were age, gender, degree of knowledge on periodontal disease, feeding level, degree of smoking, degree of oral hygiene and degree of periodontitis. Result: the prevalence of periodontal disease as a cardiovascular risk factor is $48 \%$ (19 people) with chronically moderate and advanced generalized periodontitis. Conclusion: Based on the bibliographical review people with the degree of chronic periodontitis moderate and advanced generalized are prone to trigger a cardiovascular disease (acute myocardial infarction). However, more studies of longitudinal experimentation are necessary, based on the link which has the periodontal and cardiovascular disease.

Keywords: Cardiovascular disease, acute myocardial infarction, chronic periodontitis.

\footnotetext{
* Alumna, Lic. Cirujano Dentista.

₹ Profesora Investigadora.

Universidad Juárez Autónoma de Tabasco.

Recibido: 17 de junio de 2019. Aceptado: 17 de noviembre de 2020.

Citar como: Martínez BKE, Bulnes LRM, González AM. Prevalencia de periodontitis crónica moderada y avanzada generalizada como factor de riesgo cardiovascular. Rev ADM. 2021; 78 (1): 22-27. https://dx.doi.org/10.35366/98383
} 


\section{INTRODUCCIÓN}

L a periodontitis es una enfermedad infecciosa e inflamatoria crónica, multifactorial y compleja que afecta a los tejidos de soporte y protección del diente, en términos generales es el resultado del desequilibrio entre la interacción inmunológica del huésped y la flora de la placa dentobacteriana, se ha relacionado con diferentes complicaciones sistémicas, entre ellas la enfermedad cardiovascular. ${ }^{1-3}$ La periodontitis es significante debido a que puede causar la pérdida de dientes; sin embargo, toda periodontitis se inicia con la presencia de una gingivitis y compromete el habla, reduce la calidad de vida y es una carga creciente para la economía. La manifestación principal de la periodontitis es el sangrado. ${ }^{4-6}$ En el mundo, las enfermedades cardiovasculares son la causa más común de muerte de adultos y se caracterizan por tener factores de riesgo agudo y crónico, como la aterosclerosis, que es un factor de riesgo crónico y la trombosis se comporta como un factor de riesgo agudo, en la cual se genera una obstrucción de los vasos sanguíneos que los deja en isquemia para finalmente producir un infarto cardiaco. Según la OMS, la enfermedad cardiovascular es la causa del 20\% de las muertes de la población mundial, en lo que respecta las principales causas de muerte a partir de los 40 años en los hombres y de los 64 en las mujeres. Se presenta en cuatro de cada 1,000 hombres entre los 35 y 44 años, en 10 de cada 1,000 entre los 45 y 54 años y en 20 personas de cada 1,000 entre los 55 y 64 años, y causa el $70 \%$ de los fallecimientos a partir de los 75 años. Las enfermedades periodontales y cardiovasculares comparten factores de riesgo, como la edad, tabaco, estrés, estatus socioeconómico e higiene bucal. ${ }^{7-9}$ La patogenia se debe a bolsas periodontales donde se encuentra gran cantidad de bacterias gramnegativas anaerobias que entran en contacto con el tejido subyacente, lo que favorece el paso de bacterias hacia la circulación sanguínea, produciendo bacteriemias transitorias; durante las bacteriemias se han encontrado más de 30 especies bacterianas, siendo predominantes los estreptococos viridans, responsables de patologías como endocarditis bacteriana. Estudios epidemiológicos han demostrado asociación entre periodontitis, con una mayor incidencia de eventos coronarios, Stefano investigó la relación entre el estado periodontal de personas asintomáticas con las tasas de morbilidad cardiovascular en un seguimiento de 14 años. Encontraron que los sujetos con

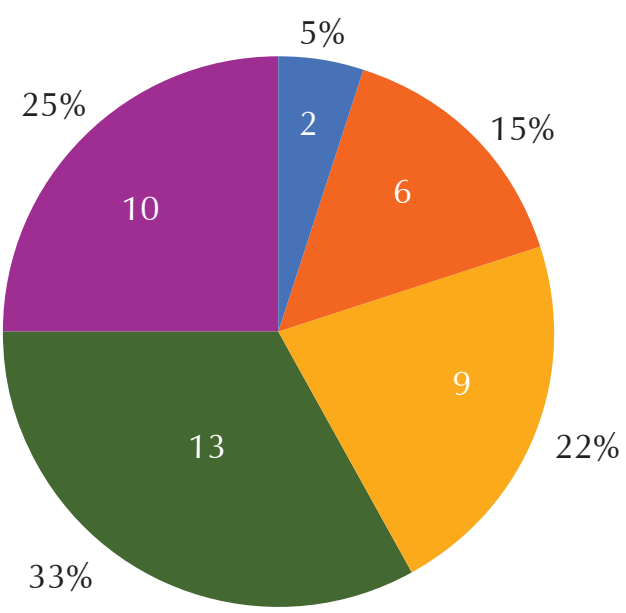

Código 0 sano

Código 1 sangrado

Código 2 cálculo

Código 3 bolsa P. 4-5 mm

Código 4 bolsa periodontal $6 \mathrm{~mm}$ o más

Figura 1: Índice periodontal comunitario.

Tabla 1: Prevalencia de enfermedad periodontal.

\begin{tabular}{llccccc}
$\begin{array}{l}\text { Índice periodontal } \\
\text { comunitario }\end{array}$ & Sano & Sangrado & Cálculo & $\begin{array}{c}\text { Bolsa periodonal } \\
4-5 \mathrm{~mm}\end{array}$ & $\begin{array}{c}\text { Bolsa periodontal } \\
6 \text { mm o más }\end{array}$ & Total \\
\hline $\begin{array}{l}\text { Género n (\%) } \\
\text { Masculino }\end{array}$ & $0(0)$ & $1(2.5)$ & $5(12.5)$ & $9(22.5)$ & $6(15)$ & $21(52.5)$ \\
$\quad$ Femenino & $2(5)$ & $5(12.5)$ & $4(10.0)$ & $4(10.0)$ & $4(10)$ & $19(47.5)$ \\
Total & $2(5)$ & $6(15.0)$ & $9(22.5)$ & $13(32.5)$ & $10(25)$ & $40(100.0)$ \\
\hline
\end{tabular}

Fuente: Exploración intrabucal de 40 adultos de 25-60 años. Tecoluta, Nacajuca, Tabasco. 
Tabla 2: Periodontitis crónica generalizada.

\begin{tabular}{lrcc}
$\begin{array}{l}\text { Índice } \\
\text { periodontal } \\
\text { comunitario }\end{array}$ & $\begin{array}{c}\text { Periodontitis } \\
\text { moderada }\end{array}$ & $\begin{array}{c}\text { Periodontitis } \\
\text { avanzada }\end{array}$ & Total \\
\hline Género n (\%) & & & \\
Masculino & $8(42.00)$ & $5(26.00)$ & $13(68.00)$ \\
Femenino & $3(16.00)$ & $3(16.00)$ & $6(32.00)$ \\
Total & $11(58.00)$ & $8(42.00)$ & $19(100.00)$ \\
\hline
\end{tabular}

Fuente: Se observó que 19 adultos de 25-60 años del poblado Tecoluta, Nacajuca, Tabasco, presentaron periodontitis crónica generalizada.

periodontitis avanzada tenían un riesgo $25 \%$ mayor de presentar accidentes cardiovasculares que las personas sin enfermedad periodontal. Múltiples estudios sugieren un incremento en el riesgo de presentar enfermedades cardiovasculares en sujetos con periodontitis. ${ }^{10-12}$ Este estudio se llevó a cabo en un poblado donde los habitantes no cuentan con información adecuada sobre la importancia de la salud bucal y general; sin embargo, se pudo determinar la prevalencia de enfermedad periodontal como factor de riesgo cardiovascular en adultos de 25 a 60 años, poblado Tecoluta, Nacajuca, Tabasco. Los resultados encontrados muestran que la periodontitis crónica moderada (32\%) y avanzada (16\%) fueron las más frecuentes. El grado de higiene bucal observado fue regular y malo, primordialmente en el género masculino. Las defunciones por infarto agudo de miocardio registrados de enero-abril 2019 fueron 16 sujetos, de los cuales el $62.5 \%$ fueron hombres y $37.5 \%$ mujeres, relacionado a la periodontitis como factor de riesgo cardiovascular. La enfermedad periodontal, por su alta prevalencia, continuará recibiendo mucha atención de la comunidad científica por ser un factor de riesgo modificable en la prevención primaria y secundaria de enfermedades cardiovasculares. Sin embargo, son necesarios más estudios de experimentación longitudinal, con base en el vínculo que tiene la enfermedad periodontal y cardiovascular. ${ }^{13-20}$

\section{MATERIAL Y MÉTODOS}

El diseño del estudio fue observacional, prospectivo, transversal y analítico. El universo fue de 400 sujetos entre 25 a 60 años, la muestra fue de 40 personas, con sistema de muestreo no probabilístico por conveniencia.
Para los criterios de inclusión se tomaron en cuenta las personas originarias del poblado Tecoluta, Nacajuca, Tabasco, hombres y mujeres de entre 25 y 60 años, que deseen colaborar y que presenten alguna enfermedad sistémica (hipertensión, diabetes mellitus, entre otros). Los materiales utilizados para la exploración intrabucal fueron kit de exploración básica (espejo, pinza, sonda periodontal y explorador), guantes de látex, cubre bocas y gorros. Las variables fueron la edad, género, grado de conocimiento sobre enfermedad periodontal, algunos factores de riesgo de enfermedad periodontal como: el nivel de alimentación, grado de tabaquismo, grado de higiene bucal y también grado de salud periodontal. La recolección de datos se obtuvo mediante un cuestionario aplicado con 45 preguntas, formado por tres secciones: grado de conocimiento sobre enfermedad periodontal, nivel de alimentación y grado de tabaquismo. Para medir el nivel o grado de higiene bucal se utilizó el índice de higiene oral simplificado, donde se examinaron seis dientes $(16,11,26,36,31,46)$ divididos por sextantes, midiendo el grado de detritus y tártaro, obteniendo el resultado mediante la suma de ambos, conforme a los códigos establecidos durante la exploración intrabucal. De igual forma, el grado de periodontitis se estableció por medio de los códigos del índice periodontal comunitario, estableciéndolo en periodontitis leve, moderada y avanzada. Los datos de defunciones por infarto agudo de miocardio fueron obtenidos del departamento de estadística del Hospital Comunitario de Nacajuca, Tabasco. Para el procesamiento estadístico, se elaboró una base de datos en Excel, utilizando estadística descriptiva, proporción para variables cualitativas y medidas de tendencia central y de dispersión para variables cuantitativas, para la presentación de resultados se emplearon tablas, graficas circulares e histogramas.

\section{RESULTADOS}

Cuarenta sujetos participaron en este estudio, 21 hombres y 19 mujeres (Tabla 1). Se utilizó el índice periodontal comunitario (Figura 1). El número de sujetos que presentan periodontitis crónica moderada y avanzada generalizada fueron 13 hombres y seis mujeres (Tabla 2). Se puede observar que el porcentaje de periodontitis crónica moderada y avanzada generalizada como factor de riesgo cardiovascular en sujetos de $25-60$ años es $48 \%$ en el poblado Tecoluta, Nacajuca, Tabasco.

El grado excelente en conocimiento sobre enfermedad periodontal es $37 \%$, el grado regular corresponde al $23 \%$ y el grado deficiente representa el $40 \%$ de la población. 


\begin{tabular}{cllll}
\hline \multicolumn{5}{c}{ Tabla 3: Grado de higiene bucal. } \\
\hline & Buena & Regular & Mala & \multicolumn{1}{c}{ Total } \\
\hline Género n (\%) & & & & \\
Masculino & $1(2.5)$ & $12(30.0)$ & $8(20.0)$ & $21(52.5)$ \\
Femenino & $7(17.5)$ & $11(27.5)$ & $1(2.5)$ & $19(47.5)$ \\
Total & $8(20.0)$ & $23(57.5)$ & $9(22.5)$ & $40(100.0)$ \\
\hline
\end{tabular}

Fuente: Exploración intrabucal a 40 habitantes de 25-60 años del poblado Tecoluta, Nacajuca, Tabasco.

En el tipo de alimentación, la población representa el $42 \%$ saludable, el $30 \%$ necesita cambios y el $28 \%$ tiene una alimentación poco saludable. En el grado de tabaquismo, la población presenta el 5\% leve, el $32 \%$ de las personas consumieron en el pasado y el $63 \%$ nunca han consumido tabaco.

El grado de higiene bucal según género por medio del índice de higiene oral simplificado, en sujetos entre 25 a 60 años del poblado Tecoluta, Nacajuca se muestra en la Tabla 3. El impacto que tiene la higiene oral en el mundo es muy significante, el grado de higiene oral es un factor de riesgo periodontal; sin embargo, el nivel más bajo de higiene en la mayoría de los casos lo presentan los hombres. Las mujeres tienen más cuidado de su salud oral, pero tiene que ver mucho la zona, el tipo de persona, los hábitos, la ocupación, la edad, el conocimiento y la importancia que cada individuo le da a su salud oral y general (Figura 2).

La proporción de muertes por infarto agudo de miocardio registrados en el Hospital Comunitario de Nacajuca, Tabasco, enero-abril 2019 (Tabla 4) es relativamente serio. Se observó que 10 fueron hombres y seis mujeres, por lo tanto, es una cifra que impacta por ser datos de un hospital y en poco tiempo.

\section{DISCUSIÓN}

El objetivo de este estudio fue determinar la prevalencia de enfermedad periodontal (periodontitis crónica moderada y avanzada) como factor de riesgo cardiovascular, los estudios experimentales longitudinales realizados establecen que las personas con el grado de periodontitis crónica moderada y avanzada generalizada son propensas a desencadenar una enfermedad cardiovascular (infarto agudo de miocardio). ${ }^{21-28}$

El primer estudio en reportar la asociación entre periodontitis y enfermedad cardiovascular fue realizado por Mattila y colaboradores en Finlandia $1989,{ }^{29}$ que concluyó que en los sujetos con infarto del miocardio (casos) comparados con sujetos sanos (controles) presentaban una deficiente salud bucal. ${ }^{8,29-34}$

También se observó que la población carece de cocimientos sobre la enfermedad periodontal y de una enseñanza-aprendizaje de la higiene bucal. Cada persona entrevistada fue seleccionada cumpliendo con los requisitos de inclusión, así también recalco que las personas encuestadas fueron de varios tipos de ocupación (maestros, albañiles, panaderos, amas de casa, campesinos, entre otros), cada persona fue entrevistada y luego se le realizó una exploración de su cavidad oral. La edad de las personas tuvo que ver mucho en cuanto a la higiene bucal, ya que se observó que el 58\% de la población tiene un grado regular en higiene oral correspondiente a hombres y mujeres de entre 25-35 años, el 22\% cuenta con una buena higiene bucal correspondiente a sujetos de entre 36-40 años y el 20\% tiene un grado malo en higiene bucal correspondiente a personas de entre 50-60 años.

\section{CONCLUSIÓN}

La mayoría de los participantes tenía periodontitis crónica generalizada, con un nivel regular y con necesidad de cambios en su alimentación. La proporción de defunciones por infarto agudo de miocardio en este año es alarmante, ya que en tan sólo cuatro meses se registraron 16 muertes. La enfermedad cardiovascular se desarrolla con más facilidad si existen varios factores al mismo tiempo y la enfermedad periodontal juega un papel muy importante en estos casos. ${ }^{35,36}$ La enfermedad periodontal, por su alta prevalencia

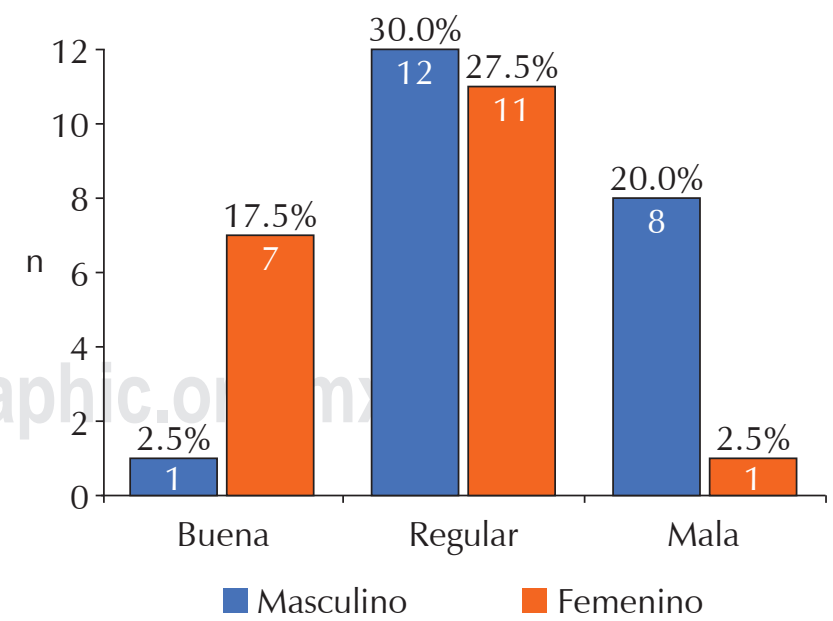

Figura 2: Grado de higiene bucal. 
Tabla 4: Defunciones por infarto de enero a abril 2019.

\begin{tabular}{cllllc} 
& Enero & Febrero & Marzo & Abril & Total \\
\hline Género n (\%) & & & & & \\
Masculino & $3(18.5)$ & $2(12.5)$ & $4(25.5)$ & $1(6.5)$ & $10(62.5)$ \\
Femenino & $1(6.5)$ & $0(0.0)$ & $2(12.5)$ & $3(18.5)$ & $6(37.5)$ \\
Total & $4(25.0)$ & $2(12.5)$ & $6(37.5)$ & $4(25.0)$ & $16(100.0)$ \\
\hline
\end{tabular}

Fuente: Base de datos obtenidos del Hospital Comunitario de Nacajuca.

mundial, continuará recibiendo mucha atención de la comunidad científica al ser un factor de riesgo modificable en la prevención primaria y secundaria de enfermedades cardiovasculares. ${ }^{9}$ Sin embargo, son necesarios más estudios de experimentación longitudinal. ${ }^{37,38}$

\section{RECOMENDACIONES}

Se sugiere plantear, en el futuro, el desarrollo de otras investigaciones que permitan medir el conocimiento que las personas poseen sobre las enfermedades cardiovasculares y realizar una comparación con otras variables consideradas como factores de riesgo cardiovascular, con mayor número de sujetos de mayor edad que los 50 años. La realización de estudios longitudinales aportaría nueva información, pues hasta la fecha no existen sobre este tema, todos son transversales.

\section{REFERENCIAS}

1. Calle CM, Ángel MP, Duque A, Giraldo A. Enfermedad periodontal y su relación con las enfermedades cardiovasculares. Rev CES Odont. 2012; 25 (1): 82-91.

2. Dietrich T, Webb I, Stenhouse L, Pattni A, Ready D, Wanyonyi $\mathrm{KL}$ et al. Evidence summary: the relationship between oral and cardiovascular disease. Br Dent J. 2017; 222: 381-385.

3. Albandar JM, Susin C, Hughes FJ. Manifestation of sistemic diseases and conditions that affects the periodontal attachment apparatus: case definitions and diagnostic considerations. J Periodontol. 2018; 45: S171-S189.

4. Leira Y, López-Dequidt I, Arias S, Rodríguez-Yáñez M, Leira R, Sobrino $T$ et al. La periodontitis crónica se asocia con infarto lacunar: un estudio de casos y controles. European Journal of Neurology. 2016; 23: 1572-1579.

5. Ljunggren S, Bengtsson T, Karlsson H, Starkhammar JC, Palm E, Nayeri $\mathrm{F}$ et al. Lipoproteínas modificadas en la periodontitis: ¿un vínculo con la enfermedad cardiovascular? Biosci Rep. 2019; 10.1042/BSR20181665.

6. Macedo Paizan ML, Vilela-Martin JF. Is there an association between periodontitis and hypertension? Curr Cardiol Rev. 2014; 10 (4): 355-361.
7. Leira Y, Seoane J, Blanco M, Rodríguez YM, Tacuache B, Blanco J, Castillo J. Asociación entre periodontitis y accidente cerebrovascular isquémico: una revisión sistemática y un metaanálisis. Revista Europea de Epidemiología. 2017; 32: 43-53.

8. Rico PJ, Pinheiro SI, Flosi CL, Partata ZE, Egbert CB, Moutinho MS. Framingham cardiovascular risk in patients with obesity and periodontitis. J Indian Soc. Periodontol. 2014; 18 (1): 14-18.

9. Sandi RM, Pol KG, Basavaraj P, Khuller N, Singh S. Association of serum cholesterol, triglyceride, high and low density lipoprotein (HDL and LDL) levels in chronic periodontitis subjects with risk for cardiovascular disease (CVD): Cross Sectional study. J Clin Diagn Res. 2014; 8 (1): 214-216.

10. Chandy S, Joseph K, Sankaranarayanan A, Issac A, Babu G, Wilson B et al. Evaluación de la proteína $C$ reactiva y fibrinógeno en pacientes con periodontitis crónica y agresiva: un estudio clínico-bioquímico. J Clin Diagn Res. 2017; 11 (3): ZC41-ZC45.

11. Natto ZS, Hameedaldain A. Evaluación de la calidad metodológica de los metaanálisis y revisiones sistemáticas de la relación entre las enfermedades periodontales y sistémicas. J Evid Based Dent Pract. 2019; 19 (2): 131-139. doi: 10.1016/j.jebdp.2018.12.003.

12. Arbes SJ, Slade GD, Beck JD. Asociación entre el alcance de la pérdida de apego periodontal y la historia autoinformada de ataque cardiaco: un análisis de datos de NHANES III. Journal of Dental Research. 10.1177 / 00220345990780120301.2016 ; 78 12: 1777-1782.

13. Khosravi SM, Jalali F, Seyyed Ahadi S, Reza HS, Dabbagh SF. The relationship between acute myocardial infarction and periodontitis. Caspian J Intern Med. 2013; 4 (2): 667-671.

14. Sarmiento LR, Velosa J, Arango D, Villegas M, Latorre C, Escobar F. Relación entre la presencia de enfermedad periodontal y el infarto agudo al miocardio por medio de la proteína $C$ reactiva ultrasensible: primera parte. Univ Odontol. 2015; 34 (73): 139-148.

15. Contreras A, Moreno S, Jaramillo A, Peláez M, Duque A, Botero JE et al. Periodontal microbiology in Latin America. Periodontology. 2015; 67 (1): 58-86.

16. David BG, Jung KJ, Mok Y, Lee SJ, Back JH, Lee S et al. Salud oral y posterior enfermedad coronaria: estudio de cohorte de un millón de personas. European Journal of Preventive Cardiology. 2018; 25 (6): 598-605. doi: 10.1177/2047487318759112.

17. Vedin O, Angstrom E, Gallu D, Neely ML, Stewart R, Koenig W et al. Periodontal disease in patients with chronic coronary heart disease: prevalece and as-association with cardiovascular risk factors. Eur J Prev Cardiol. 2015; 22 (6): 771-778.

18. Angelovich TA, Hearps AC, Jaworowsky A. Inflammation-induces foam cell Formation in chronic inflammatory disease. Immune Cell Biol. 2015; 93 (8): 683-693.

19. Ramírez J, Parra B, Gutiérrez S, Arce R, Jaramillo A, Ariza Y et al. Bio-markers of cardiovascular disease are increased in untreated chronic periodontitis: a case control study. Aust Dent J. 2014; 59 (1): 29-36.

20. Leite ACE, de Araújo $V$, Machado M. Effects of periodontal therapy on C-reactive protein and HDL in serum of subjects with periodontitis. Rev Bras Cir Cardiovasc. 2014; 29 (1): 69-77.

21. Suresh S, Narayana S, Jayakumar P, Sudhakar U, Pramod V. Evaluation of antiinflammatory effects of statins in chronic periodontitis. Indian J Pharmacol. 2013; 41 (4): 391-394.

22. Brito L, DalBo'S, Striechen T, Farias J, Olchanheski Jr. L, Mendes $\mathrm{R}$ et al. Experimental periodontitis promotes transient vascular inflammation and en-dothelial dysfunction. Arch Oral Biol. 2013; 58 (9): 1187-1198.

23. Telen MJ. Cellular adhesión and the en-dothelium E-selectin, L-Selectin, and Pan-selectin inhibitors. Hematol Oncol Clin N Am. 2014; 28: 341-354. 
24. Ramesh A, Thomas B, Amitha R. Evaluation of the association between chronic periodontitis and acute coronary syndrome: a case control study. J Indian Soc Periodontol. 2013; 17 (2): 210-213.

25. Holtfreter B, Empen K, Glaser S, Lorbeer R, Volzke H, Ewert R et al. Periodontitis is associated with endothelial dysfunction in a general population: a crosssectional study. PLoS One. 2013; 8 (12): e84603.

26. Eberhard J, Grote K, Luchtefeld M, Heuer W, Schuett H, Divchev D et al. Experimental gingivitis induces systemic inflammatory markers in young healthy individuals: a single-subject interventional study. Plos One. 2013; 8 (2): e55265.

27. Maruhashi T, Soga J, Fujimura N, Idei N, Mikami S, Iwamoto $\mathrm{Y}$ et al. Relationship between flow-mediated vasodilation and cardiovascular risk factors in a large community-based study. Heart. 2013; 99: 1837-1842.

28. Herrera C, Arcos J. Relación entre la enfermedad periodontal y la enfermedad cardiovascular: una revisión de tema. Rev Estomatol. 2012; 20 (2): 4552-4554.

29. Mattila KJ, Nieminen HS, Val tomen V. Association between dental health and acute myorcardial infactation. Br Med J. 1989; 298: 779-781.

30. López NJ, Chamorro A, Llancaqueo M. Association between atherosclerosis and periodontitis. Rev Med Chile. 2011; 139 (6): 717-724.

31. Yin G, Ryan ME. Presentación de la enfermedad periodontal: causas, patogénesis y características. En: Enfermedad periodontal y salud general: una guía para el clínico. Bogotá: Colgate; 2010. p. 4-21.

32. Thakare KS, Deo V, Bhongade ML. Evaluation of the C-reactive protein serum levels in periodontitis patients with or without atherosclerosis. Indian J Dent Res. 2010; 21 (3): 326-329.
33. Sanz M, D'Aiut F, Deanfield J, Fernandez-Aviles F. European workshop in periodontal health and cardiovascular diseaseScientific evidence on the association between periodontal and cardiovascular diseases: a review of the literature. Eur Heart J Suppl. 2010; 12 (Suppl B): B3-B12.

34. Botero JE, Bedoya E. Determinantes del diagnóstico periodontal. Rev Clin Periodon Implantol Rehabil Oral. 2010; 3 (2): 94-99.

35. Remy H, Blanchaert JR. Ischemic Heart disease. Oral Surg Oral Med Oral Pathol Oral Radiol Endod. 1999; 87: 281-283.

36. National Institute of Dental and Craniofacial Research National Oral Health Information Clearinghouse $1 \mathrm{NOHIC}$ Way Bethesda, MD 2005. pp. 92-3500, 1-866-232-4528.

37. Mattila KJ, Valtonen VV, Nieminen M, Huttunen JK. Dental infection and the risk of new coronary events: prospective study of patients with documented coronary artery disease. Clin Infect Dis. 1995; 20 (3): 588-592.

38. Rosado AA, Hernández MG, Pérez GR. Evidencias científicas de la relación entre periodontitis y enfermedades cardiovasculares. Av Periodon Implantol. 2008; 20 (3): 173-181.

\author{
Correspondencia: \\ Keyla Esmeralda Martínez Benítez \\ E-mail: keylabenitez497@gmail.com
}

Conflicto de intereses: Los autores declaran no tener ningún conflicto de intereses. 